\title{
Photon counting of extreme ultraviolet high harmonics using a superconducting nanowire single-photon detector
}

\author{
Silvio Fuchs $s^{1,2}$ (1) Johann J. Abel ${ }^{1,2} \cdot$ Jan Nathanael ${ }^{1,2} \cdot$ Julius Reinhard $^{1,2} \cdot$ Felix Wiesner $^{1,2} \cdot$ Martin Wünsche $^{1,2}$. \\ Slawomir Skruszewicz ${ }^{1,2}$. Christian Rödel ${ }^{3}$. Detlef Born ${ }^{4} \cdot$ Heidemarie Schmidt ${ }^{4,5} \cdot$ Gerhard G. Paulus $^{1,2}$
}

Received: 12 July 2021 / Accepted: 5 January 2022 / Published online: 22 January 2022

(c) The Author(s) 2022

\begin{abstract}
Laser-driven light sources in the extreme ultraviolet range (EUV) enable nanoscopic imaging with unique label-free elemental contrast. However, to fully exploit the unique properties of these new sources, novel detection schemes need to be developed. Here, we show in a proof-of-concept experiment that superconducting nanowire single-photon detectors (SNSPD) can be utilized to enable photon counting of a laser-driven EUV source based on high harmonic generation (HHG). These detectors are dark-count free and accommodate very high count rates—a perfect match for high repetition rate HHG sources. In addition to the advantages of SNSPDs for classical imaging applications with laser-driven EUV sources, the ability to count single photons paves the way for very promising applications in quantum optics and quantum imaging with high energetic radiation like, e.g., quantum ghost imaging with nanoscale resolution.
\end{abstract}

\section{Introduction}

The ability to visualize small features down to the nanoscale has ever since been an important key to scientific and technological advances. The resolution of a conventional microscope is limited by Abbe's law to approximately half of the wavelength of the light source. Thus, a straightforward way to improve the resolution is to decrease the wavelength of the light. Using light in the extreme ultraviolet (10-124 nm wavelength) and soft X-ray range (1-10 nm) for microscopy enables nanoscale resolution and exhibits an unique high elemental contrast combined with the ability to penetrate a few micrometer into solid samples [1, 2]. However, the technical realization of such microscopes is extremely

Silvio Fuchs

silvio.fuchs@uni-jena.de

1 Institute of Optics and Quantum Electronics, Friedrich Schiller University Jena, Jena, Germany

2 Helmholtz Institute Jena, Jena, Germany

3 Institute of Nuclear Physics, Technical University of Darmstadt, Darmstadt, Germany

4 Leibniz Institute of Photonic Technology (IPHT), Jena, Germany

5 Institute of Solid State Physics, Friedrich Schiller University Jena, Jena, Germany demanding in every aspect starting from the light source via the available optics to the detection of the radiation.

For a long time, synchrotrons were the only source with adequate photon flux for extreme ultraviolet (EUV) and soft X-ray (SXR) imaging applications. However, recent advances in the development of high-power ultrashort lasers drastically improved the photon-flux of laser-driven EUV sources using the process of high harmonic generation (HHG) in gases [3-5]. When ultrashort infrared laser pulses are focused into diluted gases, a small fraction of the infrared photons is coherently converted $[6,7]$ into higher harmonics of the fundamental frequency, reaching wavelengths down to a few nanometers. The emitted EUV or SXR radiation has laser-like properties such as high spatial coherence and low beam divergence. Because of the large bandwidth it can even support pulse lengths on the attosecond scale [8]. To date, HHG, at least in the low energetic EUV range $(\sim 30 \mathrm{eV})$, reaches photon-fluxes that are comparable to large-scale facility synchrotrons [9]. As a consequence, laboratory-based EUV imaging [10,11], as well as spectroscopic applications $[12,13]$ became feasible triggering widespread research activities in these fields.

Besides the light source, the technical implementation of the optical setup is equally demanding. Due to strong absorption, refractive optics like lenses cannot be used. Instead, grazing incidence mirrors or Fresnel zone plates must be employed. This, however, constrains the achievable 
numerical apertures (NA) and in consequence the resolution. These limitations can be mitigated by lensless imaging techniques $[14,15]$. The sample is illuminated by the source and the diffracted light is directly sent to the detector without any optics in between the sample and detector. Then, the actual image needs to be computed from the recorded diffraction pattern by numerically reconstructing the missing phase with sophisticated phase retrieval algorithms [16]. Since these regularly require a high degree of coherence, HHG sources are very well adapted to lensless imaging approaches [11].

Yet, one challenge remains: the detection process of the diffracted EUV radiation itself. The sensitivity of an EUV detector for lensless imaging methods should be as high as possible for several reasons. On the one hand, the resolution directly scales with the number of detected photons [17], i.e, the signal-to-noise ratio (SNR). Typically in EUV imaging, high SNR is realized by high photon flux, high quantum efficiency, and long exposure times over which the photon flux is integrated. On the other hand, it is beneficial to actually limit the incidence flux on the sample. After all, EUV and SXR light is ionizing radiation and induces damages [18].

In common EUV/SXR imaging applications typically back-illuminated thinned silicon-based CCD detectors are used as detectors. The quantum efficiency of these detectors can reach values higher than $90 \%$ [19] in the EUV range. However, the SNR is limited by read-out noise and dark counts and thus not ideal for EUV imaging with minimized photon throughput. Furthermore, due to its integrating measurement principle in conjunction with the vastly different illumination of different regions on the detector as typical for lensless imaging, these detectors cannot exploit the very high repetition rate of several $100 \mathrm{kHz}$ of high-flux HHG sources since the readout times are typically in the order of milliseconds to even seconds. Therefore, an optimal detector for imaging application with HHG radiation should offer high readout speed, ideally event-based after every laser pulse, and a high SNR, only limited by the photon shot-noise. Fast single-photon counting detectors can fulfill these requirements [20]. In addition, such detectors would open up novel quantum optical imaging possibilities in the EUV, which can reduce the required flux on the sample even below the classical limits by methods like quantum ghost imaging [21].

Photon counting in the EUV and SXR range usually has been implemented in the past by applying electron multiplier multichannel plates (MCP). However, these devices suer from low efficiencies limited by the open area ratio of the device, high dark count rates, and limited count rates. For harder X-rays, CCD cameras can detect single photons and thus can be used as counting detectors, but the above-mentioned readout-time limits persist. For lower energy photons, counting with CCDs is not possible due to read-out noise.

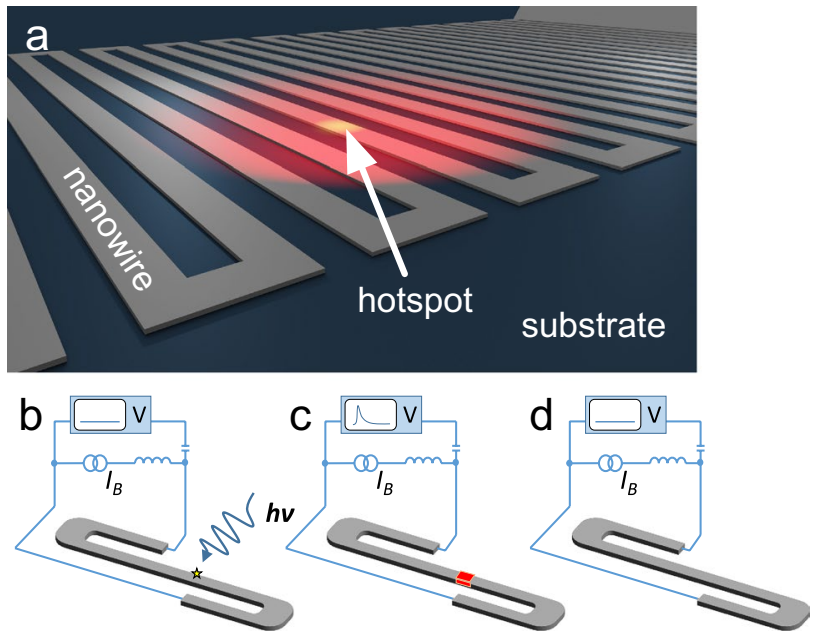

Fig. 1 SNSPD working principle in a simplified hot spot model [24, 25]: a Artwork of photon absorption by SNSPD. b Below critical current density, critical temperature and critical magnetic field, the complete meandered nanowire is superconducting and a constant DC bias current $I_{B}$ is applied. A photon with energy $h v$ hits the meander and is absorbed. c The energy of the absorbed photon is high enough to generate a so-called hot spot in the nanowire (red area). By this, the current density exceeds the critical current density of the superconductor and a small normal conducting region covers the whole crosssection of the nanowire. This resistive part together with $I_{B}$ generates a voltage peak across the SNSPD. d After a few nanoseconds (see also Fig. 3) the heat is distributed and dissipated into the substrate of the SNSPD (and finally to the cryogenic bath) and the entire nanowire becomes superconducting again and is ready for a new event

A variety of photon counting semiconducting detectors like avalanche photo diodes [22] or electron-multiplier-CCD detectors [23] are available but mostly for the infrared and optical wavelength range. Therefore, these devices typically exhibit no or very low detection efficiency for EUV and SXR photons and their count rates are typically much smaller than the repetition rate of high flux HHG sources.

Here, we demonstrate that superconducting nanowire single-photon detectors (SNSPDs) [26], which originally were also designed for infrared radiation [27], can directly be utilized for photon counting of EUV radiation from laser-driven high harmonic sources. These detectors, invented almost two decades ago [24], are based upon breaking of superconductivity in a very thin and narrow cryogenic-cooled nanowire when a photon is absorbed. Their working principle can be explained in a simplified way by the so-called hot spot model [25]: The superconducting nanowire (a few nanometers thick and tens of nanometers wide) is biased by a certain direct current (DC) $I_{B}$ below its maximum supercurrent (the critical current $I_{c}$, see Fig. 1b). If a photon with sufficiently high energy $h v$ hits the superconducting nanowire and is absorbed, a small region of the wire, the so-called hot spot, becomes normal conducting. The critical current density of the superconductor is exceeded around this hot 
spot. Therefore, the normal conducting region expands and covers the whole cross section of the nanowire (see red area in Fig. 1c). This resistive part of the nanowire together with $I_{B}$ generates a voltage peak across the SNSPD. After a few nanoseconds, the thermal energy will be distributed to the substrate of the SNSPD and finally to the cryogenic bath. The entire nanowire becomes superconducting again and the SNSPD is ready for a new photon event (Fig. 1d). The actual mechanism behind this photon detection and the role of magnetic vortices are still under investigation [28, 29]. SNSPDs can be fabricated in pixalated arrays [30], which are in principle suited for imaging applications and are predominantly used for photon counting in the far and near infrared and the visible spectral range. Nowadays, they are commercially available and become more and more popular in the heavily growing fields of quantum optics, quantum communication and quantum imaging [31-34].

When applied to EUV radiation, SNSPDs have two major advantages in comparison to other single-photon detectors like above-mentioned avalanche photo diodes or multiplier tubes. The recovery time lies in the range of a few nanoseconds or even picoseconds and thus SNSPDs can achieve count rates up to several GHz. This makes SNSPDs a perfect match to the repetition rate of state-of-the-art high-flux HHG sources driven by a repetition rate of several $100 \mathrm{kHz}$ [9]. Furthermore, SNSPDs exhibit an outstanding low dark count rate and thus would allow background-free detection. Therefore, SNSPDs in the EUV range are promising detectors not only for imaging applications, but also for experiments to measure the quantum statistics of HHG sources as Gorlach et al. proposed [35].

We want to point out that SNSPDs have actually been applied to harder $\mathrm{x}$-ray radiation $>6 \mathrm{keV}$ by Inderbitzin et al. [36-39] by increasing the thickness of the wire for higher absorption of $\mathrm{x}$-ray photons (X-SNSPD). However, to the best of our knowledge they have never been utilized neither for EUV radiation in general nor with laser-driven high harmonic sources in particular. As a proof-of-concept, we show in the following that a $10 \mathrm{~nm}$ thin meandered NbN-SNSPD, originally developed for the visible range, is capable of photon counting in the EUV.

\section{Setup}

For the proof-of-concept study a SNSPD was illuminated with EUV radiation from an HHG source [40]. Figure 2a shows the experimental setup.

The HHG source is driven by laser pulses with a central wavelength of $1300 \mathrm{~nm}$, a pulse energy of $2 \mathrm{~mJ}$, a pulse duration of $\sim 50 \mathrm{fs}$, and with a repetition rate of $1 \mathrm{kHz}$. These pulses are generated by an Optical Parametric Amplifier (OPA), which was pumped with a Ti:Sa laser (35 fs pulse duration, $9 \mathrm{~mJ}$ pulse energy central wavelength $790 \mathrm{~nm}$ ). By focusing the linearly polarized laser pulses from the OPA into an argon gas jet, the HHG process is triggered, resulting in a mixture of EUV radiation with the typical harmonic comb structure and remaining infrared laser light. EUV photons up to an energy of $\sim 100 \mathrm{eV}$ are produced [41]. They are separated from the laser radiation by thin metal foils. Two filter materials were used. Aluminum transmits EUV radiation in the range of 15 to $72 \mathrm{eV}$, whereas zirconium transmits radiation above $\sim 60 \mathrm{eV}$. In addition to the spectral filtering, the foils are also used for differential pumping of the residual gas load from the gas jet in the HHG chamber. Ultrahigh vacuum conditions at the position of the cryogenic SNSPD are crucial to avoid freezing of the nanowire chip.

The divergent EUV radiation is focused into another vacuum chamber by a toroidal mirror. At the focus of the EUV beam, a flat EUV mirror can be moved into the beam to steer it into a high resolution EUV spectrometer [42], which served as an online diagnostic for the produced HHG radiation. Without the mirror, the divergent beam propagated to another pumping stage into the detection chamber consisting of the cryogenic cooling unit and the actual SNSPD, which was exposed to the EUV radiation without any further filter foils. Due to the divergence of the EUV beam behind the focus and the finite size of the SNSPD, the amount of photon flux on the detector could be controlled by its distance to the focus. It has been set to $\sim 1 \mathrm{~m}$ to limit the photon flux in the SNSPD area to less than one photon per laser shot and thus to ensure single-photon events. The toroidal mirror was used to steer the beam to the detector and to ensure overlap.

The SNSPD consists of a $10 \mathrm{~nm}$ thick and $100 \mathrm{~nm}$ wide meandered niobium nitride nanowire with a gap size of $100 \mathrm{~nm}$, resulting in an active area of about $4.8 \times 4.8 \mu \mathrm{m}^{2}$ with a filling factor of 0.5 [27]. The nanowire is embedded in a coplanar waveguide connected to a bias-tee, which separates the bias and readout lines. A battery-powered current source was used to bias the detector. The pulses upon photon detection were amplified by means of two amplifiers (MiniCircuits ZX60-33LN-S+) at room temperature with an overall gain of about $35 \mathrm{~dB}$. The amplified signal was then observed and recorded with an oscilloscope. In addition, a standard photo diode was used to register the incoming IR-pulses on the same oscilloscope to enable coincidence measurements.

Here, we irradiate the $4.8 \times 4.8 \mu \mathrm{m}^{2}$ large active area of the SNSPD with broadband EUV radiation from an HHG source. The incident EUV spectrum is shown in Fig. 2b). It ranges from $\sim 35$ to $72 \mathrm{eV}$ limited by the aluminum filter. Hence, the detector is hit by photons with a variety of different discrete photon energies. The simulated absorption depth of the EUV radiation for three different energies $(40 \mathrm{eV}, 55 \mathrm{eV}, 70 \mathrm{eV})$ is depicted in Fig. 2c). Depending on the photon energy the absorption in the $10 \mathrm{~nm}$-thin $\mathrm{NbN}$ 


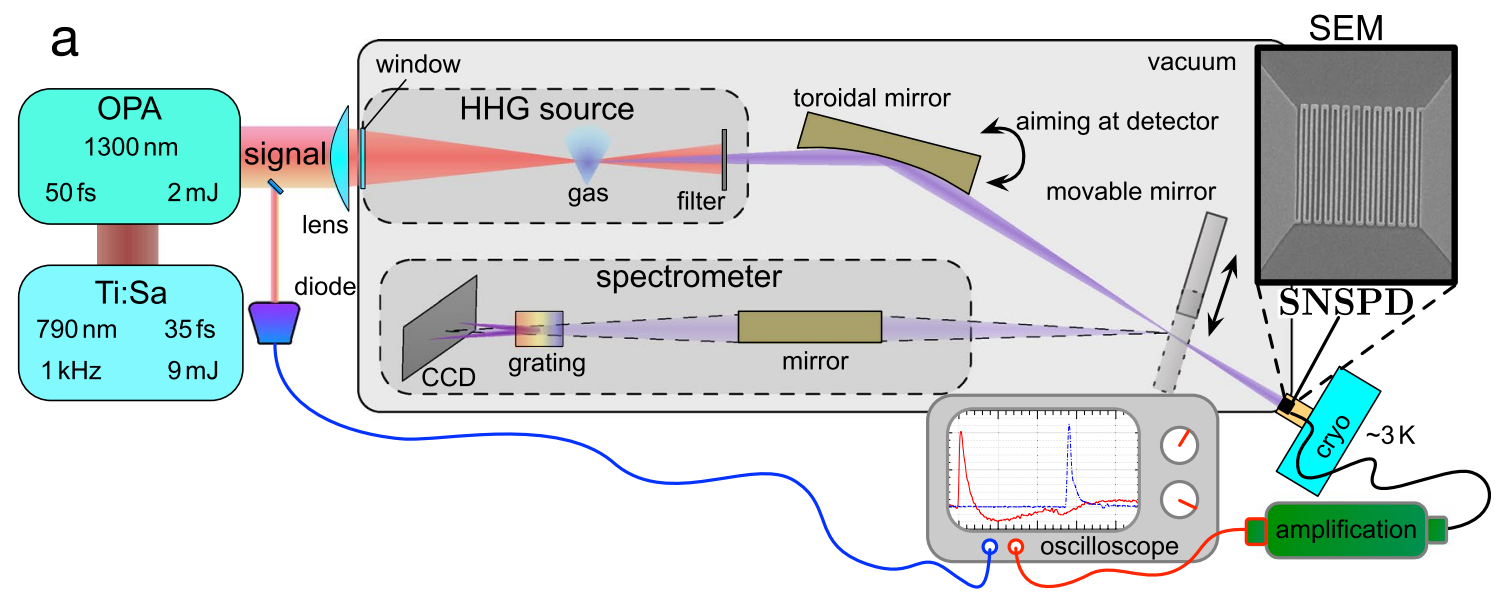

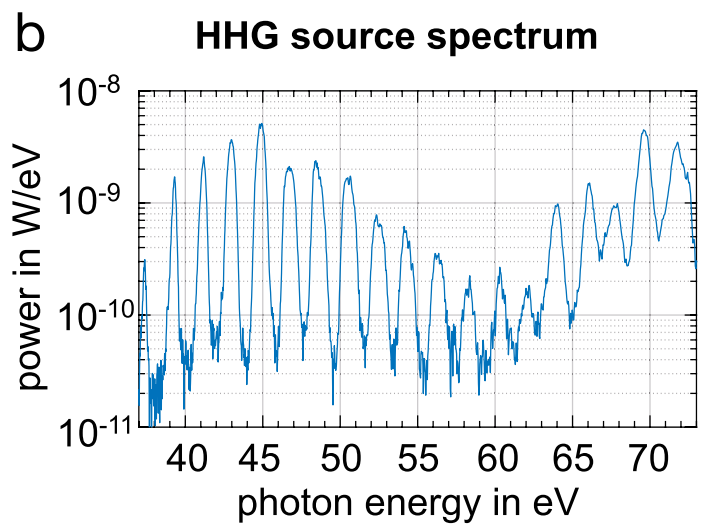

Fig. 2 a Experimental Setup: the laser radiation of an OPA is focused into an argon gas jet. EUV radiation is produced and filtered from laser light by thin metal foils. After that, the EUV beam is focused by a toroidal mirror to an intermediate focus and then propagates to the SNSPD. Additionally, a mirror can be moved to the focus area to steer the beam into an XUV spectrometer. The signal of the SNSPD is amplified with a room temperature amplifier (Mini-Circuits ZX60-33LN-S+) and measured with an oscilloscope. Furthermore, the signal of an IR diode is used to register the incoming IR pulses in coincidence. b Spectrum of the HHG radiation with an aluminum

wire is up to $35 \%$ including the filling factor of 0.5 . The remaining radiation is absorbed in the bulk material $\left(\mathrm{Al}_{2} \mathrm{O}_{3}\right)$. The absorption in the wire and thus the nominal detection efficiency could be drastically enhanced by a larger filling factor and increased wire thickness. Nevertheless, even in its current form, we demonstrate that the SNSPD is capable to count single photons of an HHG source.

\section{Results}

When the SNSPD was irradiated with the broadband EUV radiation, the detector registered single-photon events. Figure 3 shows several typical signal traces of such events at an operating temperature of the SNSPD of $3.0 \mathrm{~K}$ and a bias current of $62 \mu \mathrm{A}$ ( $74 \%$ of critical current $\left.I_{c}, I_{c}=84 \mu \mathrm{A}\right)$.

\section{EUV absorption in SNSPD}

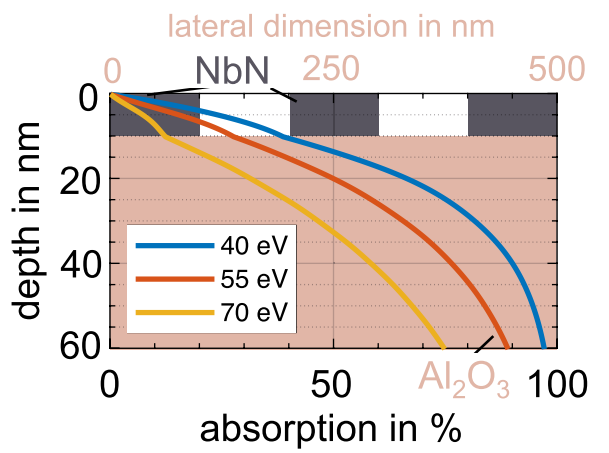

filter foil used to separate the EUV radiation from the remaining infrared laser light. The filter has a transmission window of $15-72 \mathrm{eV}$, which determines the bandwidth of the EUV photons incident on the SNSPD. c Caluculated penetration depth of EUV radiation into the SNSPD, which consists of a meandered $10 \mathrm{~nm}$ thick NbN layer on a sapphire substrate. Up to $35 \%$ of the radiation is directly absorbed in the $\mathrm{NbN}$ layer. The remaining part is absorbed in the substrate. The area lling factor of the meandered wire of 0.5 has been already included in this calculation

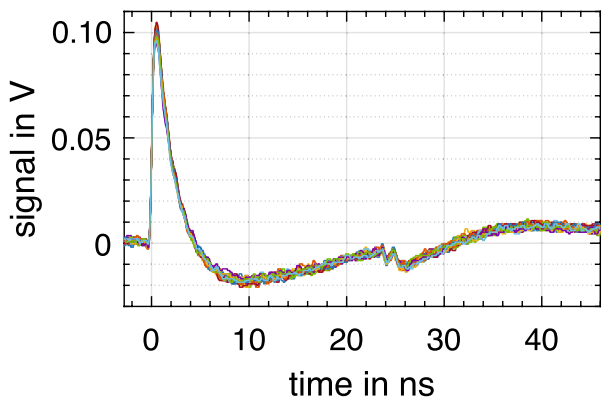

Fig. 3 Typical signal traces of the SNSPD in case of EUV photon illumination. In total 23 event traces are shown in the graph. The pulse height is determined by the operating current $I_{B}=62 \mu \mathrm{A}$, the matching of the detector to the readout circuit and the signal amplification. The operating temperature of the SNSPD was $T=3.0 \mathrm{~K}$ 
The pulse height of around $100 \mathrm{mV}$ after amplification lies in the expected range for the readout setup and corresponds to the pulse height in the case of photon detection in the visible range. For a $10 \mathrm{~nm}$ thick detector, we expect the resistance of the normal domain after photon absorption to be much higher than the $50 \Omega$ impedance of the readout. Therefore, the signal amplitude does not vary with the normal domain resistances induced by the absorption of photons of different energies. Thus, the pulse height itself does not allow for the energy resolution of the incoming photons. The decay time of around $4 \mathrm{~ns}$ is determined by the kinetic inductance of the nanowire, which roughly accounts to $L_{k}=\mu_{0} \lambda_{\mathrm{NbN}}^{2} l /(w d) \approx 42 \mathrm{nH}$, with the vacuum permeability $\mu_{0}$, the magnetic penetration depth $\lambda_{\mathrm{NbN}} \approx 550 \mathrm{~nm}$ $[27,31]$, and the nanowire's total length $l=110 \mu \mathrm{m}$, width $w=100 \mathrm{~nm}$ and thickness $d=10 \mathrm{~nm}$. The overshoot and long tail of the signal amplitude after $4 \mathrm{~ns}$ is related to the reactances in the bias and readout circuit and reflections in the readout line. The long tail is mostly caused by an impedance mismatch between the SNSPD-chip and the output line. It takes about 50-100 ns to fully converge the output signal of the measurement system. Note, that this is neither the recovery time of the SNSPD nor does it influence the detection efficiency or the dark count rate of the system.

At a bias current of $62 \mu \mathrm{A}$, the count rate was 940 events per minute and thus far below the repetition rate of the source of $1 \mathrm{kHz}$, which ensures that the detection events are related to single incident photons. To prove that the detected events are really caused by EUV photons, the measurement was repeated with the infrared driving laser switched off to show that the events are neither dark counts nor caused by any residual light inside the chamber. Indeed, no event occurred during five minutes. As a second test, the gas supply was switched off while maintaining the laser radiation to prove that the events are not triggered by infrared photons, which might have passed through the aluminum filter. Again, no events were detected within five minutes. Both findings prove that the events are caused by EUV photons. As an additional cross-check, we investigated the time-delay between the incoming laser pulse and a detected event. Figure 4 shows a typical signal trace as well as the signal of the photo-diode, which detects the incoming IR pulses (see Fig. 2a).

All detected events are clearly correlated to the laser pulses. The jitter of the time-delay between SNSPD $(l=110 \mu \mathrm{m}, w=100 \mathrm{~nm})$ signal and diode signal between 27 different events was 50ps (FWHM) at a mean value of $28.115 \mathrm{~ns}$, which corresponds to the lengths of the BNC cable $(\sim 12 \mathrm{~m})$ used for the diode signal. The time-delays of several events are depicted in Fig. 4b). The timing jitter is attributed to the hot spot emergence and subsequent vortex crossing upon photon absorption [43]. At higher photon energies, the exponential distribution diminishes. The recorded jitter lies
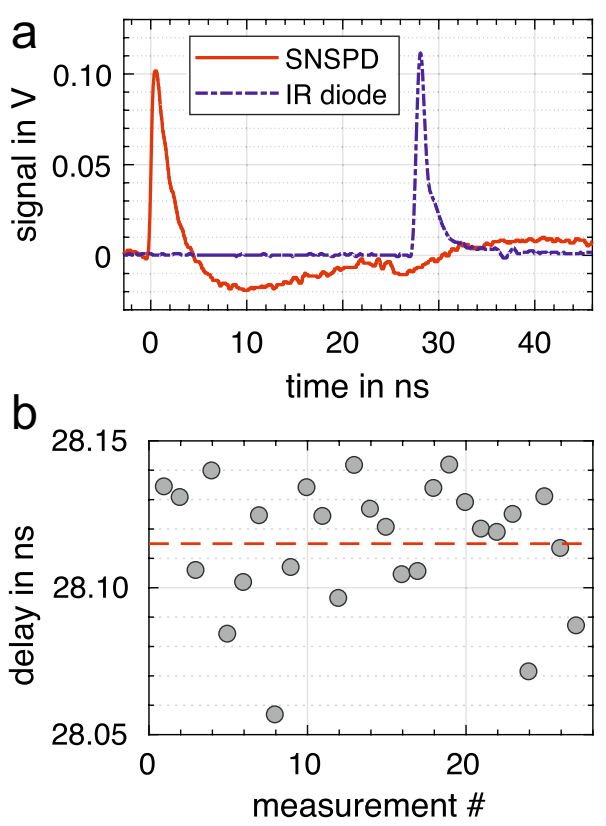

Fig. 4 a Time-delay of the EUV-related SNSPD signal to the driving infrared laser pulse: The rising edge of the SNSPD signal (red) is used for triggering. Additionally, a fast photo diode was used to detect the HHG-driving infrared laser pulse. The diode signal (blue) has been propagated within $\mathrm{a} \approx 12 \mathrm{~m}$ long $\mathrm{BNC}$ cable resulting in a delay of approx. 28ns. The bias current was set to $I_{B}=62 \mu \mathrm{A}$ and the operating temperature was $T=3.0 \mathrm{~K}$. b Time-delay between SNSPD signal and driving infrared laser pulse for several events: The gray dots depict the time-delay for a single measurement. The red dashed line shows the mean value

in the expected range, which is known from IR measurements with geometrically comparable SNSPDs $(l=30 \mu \mathrm{m}$, $w=110 \mathrm{~nm})[44]$.

After the above-mentioned proof of EUV photon detection by the SNSPD, we want to discuss the efficiency of the detection process in the following. The experiment is arranged to have a high probability to register single-photon events only. It is done in such a way, that the recorded count rate was adjusted to be significantly lower than the repetition rate of the laser. This was realized by placing the detector in the divergent beam instead of the focus region, the photon-flux hitting the detector area could be changed by adjusting the distance of the detector to the focus point. The time structure of the EUV photons within a single laser pulse is in the order of femtoseconds or even attoseconds. Thus, they are indistinguishable with respect to the time resolution of the detector, which is in the order of picoseconds. The overall flux of the source in this spectral range can be estimated to be $3 \times 10^{9}$ photons/s in the full bandwidth (see also Fig. 2b). Considering the active area of the detector of $\sim 25 \mathrm{~m}^{2}$ and the beam size $\left(1 / e^{2}\right)$ at the detector position of roughly $0.8 \mathrm{~cm}^{2}$ a maximum of 880 photons/s will hit the detector assuming an 
ideal Gaussian beam shape and perfect alignment of the small detector area directly at the center of the beam. However, as mentioned above, the measured count rate was 940 counts $/ \mathrm{min}$ or $\sim 16 \mathrm{counts} / \mathrm{s}$. This can be explained by two effects. On the one hand, the real beam shape is distorted and no longer Gaussian since a toroidal mirror (see Fig. 2a) was used for an intermediate focusing of the beam as well as for steering the beam to the fixed detector position. By aiming with the toroidal mirror to the detector, the angles are adjusted and the former Gaussian beam profile becomes strongly elongated at the detector due to astigmatism. We roughly estimate this effect to lower the incident photon number by at least one order of magnitude. On the other hand, only a part of the incident photons is actually detected by the SNSPD due to the restricted efficiency. It is limited by the absorption in the nanowire and lies around $10-35 \%$ for the given spectral window (see Fig. 2c). However, as literature predicts, it is possible to absorb a high energetic photon also in the substrate and afterwards dissipate the energy into the nanowire and still break superconductivity [36]. Consequently, this effect can increase the efficiency of the used detector in a trade-off with the timing jitter, since the duration of the dissipation process is randomly distributed. Due to the small numbers of measurements within this first proof-of-concept experiment, a robust analysis of the delay times with regard to substrate absorption is not possible. It will be investigated in continuative experiments.

Nanowires with thicknesses in the range of the penetration depth of EUV photons into $\mathrm{NbN}$ would ensure that the photons are absorbed by the SNSPD and not by the underlying substrate. This will significantly increase the overall detection efficiency of the detector from which the targeted EUV imaging applications would heavily benefit. Therefore, SNSPDs can be optimized for the EUV range as they have been developed for the $x$-ray range [37].

To quantify the effect of the bias current on the detection rate of the SNSPD, the count rates for different currents were measured by keeping all other parameters constant. The result is depicted in Fig. 5.

With increased bias current the count rate is also increased. This effect can be explained by the spectral bandwidth of the HHG radiation. The detector is illuminated with broadband harmonic radiation between $\sim 35-72 \mathrm{eV}$. For a low bias current only photons with very high energies are able to break superconductivity and thus are registered. In other words, the detector is blinded for all photons below a certain energy depending on the bias current. For higher bias currents this detection limit shifts to lower photon energies. This gives rise to a certain integrated energy resolution of the detection scheme by altering the bias current. Spectrallyresolved measurements will be conducted in the future to investigate this effect in more detail.

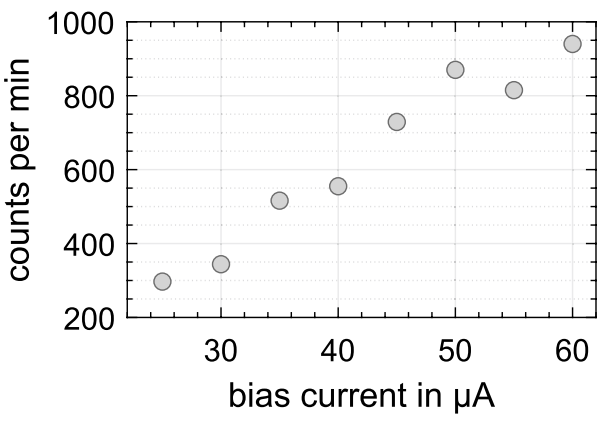

Fig. 5 Count rate of the SNSPD $\left(I_{c}=84 \mu \mathrm{A}, T=3.03 \mathrm{~K}\right)$ in dependence of the bias current

The HHG source is capable of producing photons up to an energy of $\sim 100 \mathrm{eV}$. To demonstrate the capabilities of the SNSPD in this higher energetic region, we interchanged the aluminum filter of the HHG source with a zirconium foil. Thus, radiation between $\sim 60-100 \mathrm{eV}$ hits the detector and could be registered. However, the IR-transmission of the used zirconium foils for the driving laser wavelength was higher in comparison to the aluminum filter. Therefore, infrared photons reached the detector and caused high count rates in the order of 20counts per second even without actual harmonic radiation. By exploiting the above-mentioned dependence of the detection process on the bias current it was however possible to select a current $\left(I_{B}=30 \mu \mathrm{A}\right)$, which switched the sensitivity of the SNSPD for infrared photons almost completely off while simultaneously maintaining the EUV sensitivity. Therefore, the detector can be used to count EUV photons even if remaining infrared laser light is present. The ability to blind the detector for IR light is a significant advantage over other detector technologies. To preserve the detector from too high infrared photon-flux, we had to slightly changed the beam direction for measurements at higher photon energies to hit the detector only at the edge of the Gaussian light distribution. For this reason, we measured an EUV count rate of 5 counts/minute for EUV photons above $60 \mathrm{eV}$. The background count rate with infrared laser only (gas supply off) was 0.6 counts/minute.

\section{Conclusion}

Our findings prove that a single-photon detector based on superconducting nanowires can be utilized for photon counting of EUV photons in particular from a laser-driven HHG source with very low dark count rates. The laser repetition rate in the presented experiment is $1 \mathrm{kHz}$. Novel fiber-laser approaches for high-power laboratory-based HHG sources however are realized by drastically increasing the repetition rate up to the $\mathrm{MHz}$ [45] regime so that the high possible count-rate of the SNSPD can be exploited in the future. The 
detector can be blinded for infrared photons by adjusting the bias current, which is a major advantage for laser-based EUV sources. Apart from the possibility to disregard low energy photons, SNSPDs inherently offer only limited ways to differentiate between photons of different energies [46].

For imaging purposes, spatial resolution needs to be added to the detection scheme. This could be implemented with pixelated setups of several SNSPDs or by measuring the position of the event on the meandered wire by evaluating the delay between the signal at the two ends [47]. The presented proof-of-concept paves the way for future applications of SNSPDs and other cryogenic detector schemes [48, 49] for laser-based high harmonic light sources in coherent imaging [50], EUV quantum optics [35], and quantum imaging $[51,52]$.

Acknowledgements The authors thank Emanuel Knehr for his help with the SNSPD and fruitful discussions and Mario Ziegler for the production of the SNSPD.

Funding Open Access funding enabled and organized by Projekt DEAL. Thüringer Aufbaubank (Forschergruppen 2015FGR0094 and 2018FGR0080 and Quantum Hub 2021FGI0049); Helmholtz Institute Jena; German Federal Ministry for Education (BMBF) (VIP "X-CoherenT"); AppQuant (ZF4006821DF9); German Research Foundation FG IR-TG Meta Active (DFG-RTG 2675). Felix Wiesner is part of the Max Planck School of Photonics supported by BMBF, the Max Planck Society, and the Fraunhofer Society.

Open Access This article is licensed under a Creative Commons Attribution 4.0 International License, which permits use, sharing, adaptation, distribution and reproduction in any medium or format, as long as you give appropriate credit to the original author(s) and the source, provide a link to the Creative Commons licence, and indicate if changes were made. The images or other third party material in this article are included in the article's Creative Commons licence, unless indicated otherwise in a credit line to the material. If material is not included in the article's Creative Commons licence and your intended use is not permitted by statutory regulation or exceeds the permitted use, you will need to obtain permission directly from the copyright holder. To view a copy of this licence, visit http://creativecommons.org/licenses/by/4.0/.

\section{References}

1. D.A. Shapiro, Y.-S. Yu, T. Tyliszczak, J. Cabana, R. Celestre, W. Chao, K. Kaznatcheev, A.L.D. Kilcoyne, F. Maia, S. Marchesini, Y.S. Meng, T. Warwick, L.L. Yang, H.A. Padmore, Chemical composition mapping with nanometre resolution by soft X-ray microscopy. Nat. Photon. 8, 765-769 (2014)

2. F. Wiesner, M. Wünsche, J. Reinhard, J.J. Abel, J. Nathanael, S. Skruszewicz, C. Rödel, S. Yulin, A. Gawlik, G. Schmidl, U. Hübner, J. Plentz, G.G. Paulus, S. Fuchs, Material-specific imaging of nanolayers using extreme ultraviolet coherence tomography. Optica 8, 230 (2021)

3. E. Constant, D. Garzella, P. Breger, E. Mével, C. Dorrer, C. Le Blanc, F. Salin, P. Agostini, Optimizing high harmonic generation in absorbing gases: model and experiment. Phys. Rev. Lett. 82, 1668-1671 (1999)
4. H. Carstens, M. Högner, T. Saule, S. Holzberger, N. Lilienfein, A. Guggenmos, C. Jocher, T. Eidam, D. Esser, V. Tosa, V. Pervak, J. Limpert, A. Tünnermann, U. Kleineberg, F. Krausz, I. Pupeza, High-harmonic generation at $250 \mathrm{MHz}$ with photon energies exceeding 100 eV. Optica 3, 366 (2016)

5. S. Hädrich, A. Klenke, J. Rothhardt, M. Krebs, A. Hoffmann, O. Pronin, V. Pervak, J. Limpert, A. Tünnermann, High photon flux table-top coherent extreme-ultraviolet source. Nat. Photon. 8, 779-783 (2014)

6. J.L. Krause, K.J. Schafer, K.C. Kulander, High-order harmonic generation from atoms and ions in the high intensity regime. Phys. Rev. Lett. 68, 3535-3538 (1992)

7. P.B. Corkum, Plasma perspective on strong field multiphoton ionization. Phys. Rev. Lett. 71, 1994-1997 (1993)

8. P.B. Corkum, F. Krausz, Attosecond science. Nat. Phys. 3, 381387 (2007)

9. R. Klas, S. Demmler, M. Tschernajew, S. Hädrich, Y. Shamir, A. Tünnermann, J. Rothhardt, J. Limpert, Table-top milliwatt-class extreme ultraviolet high harmonic light source. Optica 3, 1167 (2016)

10. M.D. Seaberg, B. Zhang, D.F. Gardner, E.R. Shanblatt, M.M. Murnane, H.C. Kapteyn, D.E. Adams, Tabletop nanometer extreme ultraviolet imaging in an extended reflection mode using coherent Fresnel ptychography. Optica 1, 39 (2014)

11. M. Zürch, J. Rothhardt, S. Hädrich, S. Demmler, M. Krebs, J. Limpert, A. Tünnermann, A. Guggenmos, U. Kleineberg, C. Spielmann, Real-time and sub-wavelength ultrafast coherent diffraction imaging in the extreme ultraviolet. Sci. Rep. 4, 7356 (2014)

12. D. Popmintchev, B.R. Galloway, M.-C. Chen, F. Dollar, C.A. Mancuso, A. Hankla, L. Miaja-Avila, G. O’Neil, J.M. Shaw, G. Fan, S. Ališauskas, G. Andriukaitis, T. Balčiunas, O.D. Mücke, A. Pugzlys, A. Baltuška, H.C. Kapteyn, T. Popmintchev, M.M. Murnane, Near- and extended-edge X-ray-absorption fine-structure spectroscopy using ultrafast coherent high-order harmonic supercontinua. Phys. Rev. Lett. 120, 093002 (2018)

13. B. Buades, D. Moonshiram, T.P.H. Sidiropoulos, I. León, P. Schmidt, I. Pi, N. Di Palo, S.L. Cousin, A. Picón, F. Koppens, J. Biegert, Dispersive soft X-ray absorption fine-structure spectroscopy in graphite with an attosecond pulse. Optica 5, 502 (2018)

14. H.N. Chapman, K.A. Nugent, Coherent lensless X-ray imaging. Nat. Photon. 4, 833-839 (2010)

15. F. Pfeiffer, X-ray ptychography. Nat. Photon. 12, 9-17 (2018)

16. Y. Shechtman, Y.C. Eldar, O. Cohen, H.N. Chapman, J. Miao, M. Segev, Phase retrieval with application to optical imaging: a contemporary overview. IEEE Signal Process. Mag. 32, 87-109 (2015)

17. A. Schropp, C.G. Schroer, Dose requirements for resolving a given feature in an object by coherent $\mathrm{X}$-ray diffraction imaging. New $\mathrm{J}$. Phys. 12, 035016 (2010)

18. B. Ziaja, H.N. Chapman, R. Fäustlin, S. Hau-Riege, Z. Jurek, A.V. Martin, S. Toleikis, F. Wang, E. Weckert, R. Santra, Limitations of coherent diffractive imaging of single objects due to their damage by intense X-ray radiation. New J. Phys. 14, 115015 (2012)

19. I. Moody, M. Watkins, R. Bell, M. Soman, J. Keelan, A. Holland, CCD QE in the Soft X-ray Range. (2017)

20. L. You, Superconducting nanowire single-photon detectors for quantum information. Nanophotonics 9, 2673-2692 (2020)

21. M. Gilaberte Basset, F. Setzpfandt, F. Steinlechner, E. Beckert, T. Pertsch, M. Gräfe, Perspectives for applications of quantum imaging. Laser Photon. Rev. 13, 1900097 (2019)

22. S. Cova, M. Ghioni, A. Lacaita, C. Samori, F. Zappa, Avalanche photodiodes and quenching circuits for single-photon detection. Appl. Opt. 35, 1956 (1996) 
23. D. J. Denvir, E. Conroy, Electron-multiplying CCD: the new ICCD. In Proc. SPIE, vol. 4796 C. B. Johnson, D. Sinha, P. A. Laplante, eds. (2003), p. 164

24. G.N. Gol'tsman, O. Okunev, G. Chulkova, A. Lipatov, A. Semenov, K. Smirnov, B. Voronov, A. Dzardanov, C. Williams, R. Sobolewski, Picosecond superconducting single-photon optical detector. Appl. Phys. Lett. 79, 705-707 (2001)

25. A.D. Semenov, G.N. Gol'tsman, A.A. Korneev, Quantum detection by current carrying superconducting film. Phys. C Supercond. 351, 349-356 (2001)

26. I. Esmaeil Zadeh, J. Chang, J.W.N. Los, S. Gyger, A.W. Elshaari, S. Steinhauer, S.N. Dorenbos, V. Zwiller, Superconducting nanowire single-photon detectors: a perspective on evolution, state-of-the-art, future developments, and applications. Appl. Phys. Lett. 118, 190502 (2021)

27. E. Knehr, A. Kuzmin, D.Y. Vodolazov, M. Ziegler, S. Doerner, K. Ilin, M. Siegel, R. Stolz, H. Schmidt, Nanowire single-photon detectors made of atomic layer-deposited niobium nitride. Supercond. Sci. Technol. 32, 125007 (2019)

28. A. Engel, J.J. Renema, K. Il'in, A. Semenov, Detection mechanism of superconducting nanowire single-photon detectors. Supercond. Sci. Technol. 28, 114003 (2015)

29. D.Y. Vodolazov, Single-photon detection by a dirty current-carrying superconducting strip based on the Kinetic-equation approach. Phys. Rev. Appl. 7, 034014 (2017)

30. E.E. Wollman, V.B. Verma, A.E. Lita, W.H. Farr, M.D. Shaw, R.P. Mirin, S. Woo Nam, Kilopixel array of superconducting nanowire single-photon detectors. Opt. Express 27, 35279 (2019)

31. C.M. Natarajan, M.G. Tanner, R.H. Hadfield, Superconducting nanowire single-photon detectors: physics and applications. Supercond. Sci. Technol. 25, 063001 (2012)

32. H. Takesue, S.W. Nam, Q. Zhang, R.H. Hadfield, T. Honjo, K. Tamaki, Y. Yamamoto, Quantum key distribution over a 40-dB channel loss using superconducting single-photon detectors. Nat. Photon. 1, 343-348 (2007)

33. H. Takesue, S.D. Dyer, M.J. Stevens, V. Verma, R.P. Mirin, S.W. Nam, Quantum teleportation over $100 \mathrm{~km}$ of fiber using highly efficient superconducting nanowire single-photon detectors. Optica 2, 832 (2015)

34. S. Khasminskaya, F. Pyatkov, K. Słowik, S. Ferrari, O. Kahl, V. Kovalyuk, P. Rath, A. Vetter, F. Hennrich, M.M. Kappes, G. Gol'tsman, A. Korneev, C. Rockstuhl, R. Krupke, W.H.P. Pernice, Fully integrated quantum photonic circuit with an electrically driven light source. Nat. Photon. 10, 727-732 (2016)

35. A. Gorlach, O. Neufeld, N. Rivera, O. Cohen, I. Kaminer, The quantum-optical nature of high harmonic generation. Nat. Commun. 11, 4598 (2020)

36. D. Perez de Lara, M. Ejrnaes, A. Casaburi, M. Lisitskiy, R. Cristiano, S. Pagano, A. Gaggero, R. Leoni, G. Golt'sman, B. Voronov, Feasibility investigation of $\mathrm{NbN}$ nanowires as detector in time-of-flight mass spectrometers for macromolecules of interest in biology (proteins). J. Low Temp. Phys. 151, 771-776 (2008)

37. K. Inderbitzin, A. Engel, A. Schilling, K. Il'in, M. Siegel, An ultra-fast superconducting $\mathrm{Nb}$ nanowire single-photon detector for soft X-rays. Appl. Phys. Lett. 101, 162601 (2012)

38. K. Inderbitzin, A. Engel, A. Schilling, Soft X-ray single-photon detection with superconducting tantalum nitride and niobium nanowires. IEEE Trans. Appl. Supercond. 23, 2200505-2200505 (2013)

39. X. Zhang, Q. Wang, A. Schilling, Superconducting single X-ray photon detector based on W0.8Si0.2. AIP Adv. 6, 0-7 (2016)
40. M. Wünsche, S. Fuchs, S. Aull, J. Nathanael, M. Möller, C. Rödel, G.G. Paulus, Quasi-supercontinuum source in the extreme ultraviolet using multiple frequency combs from high-harmonic generation. Opt. Express 25, 6936 (2017)

41. J. Nathanael, M. Wünsche, S. Fuchs, T. Weber, J.J. Abel, J. Reinhard, F. Wiesner, U. Hübner, S.J. Skruszewicz, G.G. Paulus, C. Rödel, Laboratory setup for extreme ultraviolet coherence tomography driven by a high-harmonic source. Rev. Sci. Instrum. 90, 113702 (2019)

42. M. Wünsche, S. Fuchs, T. Weber, J. Nathanael, J.J. Abel, J. Reinhard, F. Wiesner, U. Hübner, S.J. Skruszewicz, G.G. Paulus, C. Rödel, A high resolution extreme ultraviolet spectrometer system optimized for harmonic spectroscopy and XUV beam analysis. Rev. Sci. Instrum. 90, 023108 (2019)

43. M. Sidorova, A. Semenov, H.-W. Hübers, I. Charaev, A. Kuzmin, S. Doerner, M. Siegel, Physical mechanisms of timing jitter in photon detection by current-carrying superconducting nanowires. Phys. Rev. B 96, 184504 (2017)

44. M.A. Wolff, S. Vogel, L. Splitthoff, C. Schuck, Superconducting nanowire single-photon detectors integrated with tantalum pentoxide waveguides. Sci. Rep. 10, 17170 (2020)

45. A. Vernaleken, J. Weitenberg, T. Sartorius, P. Russbueldt, W. Schneider, S.L. Stebbings, M.F. Kling, P. Hommelhoff, H.-D. Hoffmann, R. Poprawe, F. Krausz, T.W. Hänsch, T. Udem, Single-pass high-harmonic generation at $208 \mathrm{MHz}$ repetition rate. Opt. Lett. 36, 3428 (2011)

46. A.D. Semenov, P. Haas, B. Günther, H.-W. Hübers, K. Il'in, M Siegel, Energy resolution of a superconducting nanowire singlephoton detector. J. Low Temp. Phys. 151, 564-569 (2008)

47. Q.-Y. Zhao, D. Zhu, N. Calandri, A.E. Dane, A.N. McCaughan, F. Bellei, H.-Z. Wang, D.F. Santavicca, K.K. Berggren, Singlephoton imager based on a superconducting nanowire delay line. Nat. Photon. 11, 247-251 (2017)

48. A. Peacock, P. Verhoeve, N. Rando, A. van Dordrecht, B.G. Taylor, C. Erd, M.A.C. Perryman, R. Venn, J. Howlett, D.J. Goldie, J. Lumley, M. Wallis, Single optical photon detection with a superconducting tunnel junction. Nature 381, 135-137 (1996)

49. W. B. Doriese, P. Abbamonte, B. K. Alpert, D. A. Bennett, E. V. Denison, Y. Fang, D. A. Fischer, C. P. Fitzgerald, J. W. Fowler, J. D. Gard, J. P. Hays-Wehle, G. C. Hilton, C. Jaye, J. L. McChesney, L. Miaja-Avila, K. M. Morgan, Y. I. Joe, G. C. O'Neil, C. D. Reintsema, F. Rodolakis, D. R. Schmidt, H. Tatsuno, J. Uhlig, L. R. Vale, J. N. Ullom, D. S. Swetz, A practical superconducting-microcalorimeter X-ray spectrometer for beamline and laboratory science. Rev. Sci. Instrum. 88 (2017)

50. A. Classen, K. Ayyer, H.N. Chapman, R. Röhlsberger, J. von Zanthier, Incoherent diffractive imaging via intensity correlations of hard X rays. Phys. Rev. Lett. 119, 053401 (2017)

51. A.-X. Zhang, Y.-H. He, L.-A. Wu, L.-M. Chen, B.-B. Wang, Tabletop $\mathrm{X}$-ray ghost imaging with ultra-low radiation. Optica 5, 374 (2018)

52. A. Schori, S. Shwartz, X-ray ghost imaging with a laboratory source. Opt. Express 25, 14822 (2017)

Publisher's Note Springer Nature remains neutral with regard to jurisdictional claims in published maps and institutional affiliations. 